\title{
La médecine de premier recours en chantier: service de garde et assistanat au cabinet médical
}

Le Département fédéral de l'intérieur (DFI) et la Conférence des directrices et directeurs cantonaux de la santé (CDS) ont décidé fin mars 2006, sur la base d'un document de travail [1] visant à soutenir la médecine de premier recours, de mettre sur pied un groupe de travail sous la houlette de la CDS. Des représentants des médecins cantonaux, du CMPR, de l'ASMAC, de la FMH, de santésuisse, de l'OFSP et de la CDS, séparés en deux sous-groupes, ont élaboré des propositions pour lever les impasses dans le service des urgences médicales ambulatoires et pour financer des offres spécifiques de formation postgraduée en médecine de premier recours. Le groupe de travail a adopté, le 19 septembre 2006, les rapports rédigés par le secrétariat de la CDS qui seront présentés fin octobre aux décideurs politiques.

Dans le domaine des services d'urgence médicale, ces travaux ont engendré dans toute la Suisse un processus visant à assurer l'assistance médicale d'urgence à la population tout en la rendant plus efficace. Dans des régions de taille et de densité médicale très diverses, on cherchera des solutions de partenariat entre fournisseurs de prestations ambulatoires et hospitaliers.

Dans le domaine de la formation postgraduée en médecine de premier recours, les travaux ont fait naître, même avant l'adoption du rapport, d'intéressantes activités cantonales et régionales. L'importance d'une formation postgraduée au sein du futur cadre professionnel est largement reconnue. A brève échéance, l'assistanat au cabinet médical devra être mis à égalité avec celui pratiqué dans les hôpitaux grâce au soutien financier des cantons. A moyen terme, il faudra créer des conditions qui permettent de développer un marché de la formation postgraduée bien rodé, apte à concevoir plus efficacement la formation postgraduée des médecins de premier recours. Le chantier est donc en cours. La CDS a convoqué une nouvelle fois les experts du groupe de travail: tout système de santé efficace nécessite une médecine de premier recours abondante et de qualité [2]. Il faudra concevoir la formation prégraduée et postgraduée des futurs omnipraticiens selon les besoins de la population [3], la renforcer et la rendre plus efficace pour l'ère de la médecine prédictive. La médecine de premier recours doit devenir une activité plus attrayante et être adaptée aux conditions sociales et démographiques des nouvelles générations de médecins.

Max Giger, membre du Comité central de la FMH, président de la Commission pour la formation postgraduée et continue de la FMH

1 Trutmann M. Magna cum cura. http://www.gdk-cds.ch/ fileadmin/pdf/Gesundheitsversorgung/Planung Gesversorgung/Grundversorgung/Grundversorger_III.pdf.

2 Starfield B, Shi Leiyo, Macinko J. Contribution of Primary Care to Health Systems and Health. The Milbank Quarterly. 2005;83:457-502.

3 Safran DG. Defining the Future of Primary Care: What Can We Learn from Patients? Ann Intern Med. 2003;138:248-55.

Remarque: Le rapport évoqué dans cet éditorial sera très prochainement rendu public par la CDS et diffusé sous forme résumée dans le BMS - nous reprendrons le sujet à cette occasion, en lui donnant également d'autres éclairages. 